\title{
An alternative mobility \& transportation that uses gravity for the colonization of the Moon
}

\author{
M.A. De Andrade ${ }^{10}$, L.G. Ferreira Filho ${ }^{*}$, C. Neves ${ }^{1}$ \\ ${ }^{1}$ Universidade do Estado do Rio de Janeiro, Faculdade de Tecnologia, Resende, RJ, Brasil
}

Received on November 28, 2019. Revised on February 4, 2020. Accepted on February 10, 2020.

\begin{abstract}
It is well known that hypothetical tunnels net within the Earth is usually considered as an illustration in a gravitation course for undergraduate students. In this context, an uniform mass distribution is usually considered and, consequently, a vehicle inside the tunnel oscillates in a simple harmonic motion (SHM). In this work, we investigate this problem and we argue that, instead to illustrate this problem using Earth, it is better to consider the Moon. In order to support this proposition, we present a twofold argumentation: first, we show that there is a quite discrepancy between the results obtained from Earth uniform mass distribution model with those obtained considering realistic Earth models (PREM model), whose mass is nonuniformly distributed; second, when the Moon is considered, we show that there is not a discrepancy between the results computed from uniform mass distribution model with those computed from nonuniform mass distribution. Indeed, we revealed that a vehicle inside the tunnel within Moon oscillates approximately in a SHM.
\end{abstract}

Keywords: Moon, potential energy, gravitational transport.

\section{Introduction}

The Moon colonization is still a ludic topic that drives the human spirit to embrace nobles causes and some efforts that lift the status quo of the human thought. In order to get this, the Moon colonization can play a very important role, since it can be used as a first camp base to start the exploration of the outer space. At this context, the construction of an efficient mobility \& transportation net is mandatory to support such an enterprise. A net of tunnels (chords) connecting different points on the Moon surface could use the gravitational energy of the mass distribution of the Moon. On the special case of constant density, the elapsed time in travels between two any points would be always the same, regardless the extension of the tunnel (tautochrone). The geometry of the tunnel is relevant for the elapsed time. In particular, a minimum transit time is attained in a cycloid tunnel (brachistochrone) 1].

Such a transportation method has already been suggested for the Earth 2, 3. In an idealized uniform mass distribution, a vehicle inside the tunnel would oscillate in a simple harmonic motion (SHM). In Ref. [4 the author proposed a more realistic model, in which the Earth density is considered non uniform. This geological model is constructed on the data base denominated Preliminary reference Earth model (PREM) [5]. In another reference [6] the author uses a model that takes into account astrophysical and thermodynamical concepts to improve the calculations. It is worth to say that this

*Correspondence email address: kph120@gmail.com model 7 fits very well to the stars, more specifically, to the Sun.

Inspired by this mobility \& transportation problem, we also investigate the inner structure of Moon through three Moon models. Recently 8 it was proposed a geological model for the Moon based on the data collected by the Apollo Mission, called Very preliminary reference Moon model (VPREMOON). They used this model to make some assumptions about the Moon core composition. With the same purpose, afterwards 9] it was elaborated a model with two variants. As a complement to the VPREMOON, this model regards the Moon core as a structure that can be divided in two parts: Inner core and outer core.

Since the hypothetical tunnels on Moon can be built, it is important to know how the physical quantities behave within the Moon. In order to clarify this point, we departure from those Moon models and, through some numerical computation and experimental data, numerical results for some physical quantities are calculated: time, position, velocity, acceleration and pressure on the tunnel walls. After that, we compare these kinematic results computed for each model: SHM model, VPREMOON Model and the two variants models proposed by Jing et al, which we call JING1 model and JING2 model.

The paper is organized as follows: in section 2, it is presented the free fall problem in the gravitational Moon field. In the sections 3 and 4 the VPREMOON and JING models are discussed. In the section 5 the different results for free fall problem in gravitational Moon field, based in the Moon models previously presented, are compared 
and discussed. At the end, some conclusions are pointed out.

\section{Free fall in gravitational tunnel in the Moon}

Consider a gravitational vehicle in free fall in a tunnel drilled along a diameter of the Moon. Comparing the dimensions of the gravitational vehicle with the dimensions of the Moon (the radius of the Moon is $R=1737 \mathrm{~km}$ ), the vehicle can be considered as a particle, and the analysis for the particle will also apply to the vehicle. The tunnel will pass through the center of the Moon, so it will cross all layers with different densities that exist inside the Moon. According to Newton's shell theorem, a particle immersed in a spherically symmetric distribution of mass and located at a distance $r$ from the center of this distribution, $r<R$, perceives only the gravitational forces of the mass contained in the inner sphere of radius $r$, because the gravitational forces on this particle exerted by the mass contained in the concentric shells with values of radius greater than $r$ cancel and have no effect, therefore the acceleration $a(r)$ of a body falling into the tunnel under the action of gravity must be related to the mass $M_{r}$ contained in the inner sphere whose radius coincides with the line connecting the center of the Moon to the point of the tunnel where the particle is located. The total mass of the Moon, $M_{R}$, will be denoted by $M$. From the Newton's law of universal gravitation follows that the acceleration of the free falling particle in the tunnel, whose value is identified with the acceleration of gravity at that point, is given by

$$
a(r)=-\frac{G M_{r}}{r^{2}} .
$$

The mass of the inner sphere of radius $r$ concentric to the spherical distribution can be obtained through the density $\rho(r)$ as

$$
M_{r}=4 \pi \int_{0}^{r} \rho\left(r^{\prime}\right){r^{\prime}}^{2} d r^{\prime} .
$$

We can express $M_{r}$ in terms of the density at the center of the Moon $\rho_{c}$ with the help of the function ${ }^{1} h(r)$ defined by

$$
h(r)=\frac{3}{\rho_{c} r^{2}} \int_{0}^{r}\left[\rho_{c}-\rho\left(r^{\prime}\right)\right] r^{\prime 2} d r^{\prime} .
$$

It follows that,

$$
M_{r}=\frac{4 \pi \rho_{c}}{3} r^{2}[r-h(r)] .
$$

Taking $r=R$ in the previous equation, we can express $\rho_{c}$ in terms of the mean density of the Moon $\bar{\rho}=3 M / 4 \pi R^{3}$ by

$$
\rho_{c}=\bar{\rho} \frac{R}{R-h(R)} .
$$

${ }^{1}$ For the function $h(r)$ to be mathematically well defined at $r=0$, the function $\rho(r)$ must be expressed by polynomials with lowest power equal or greater than -1 .
Therefore, from Eq. (1), Eq. (4) and Eq. (5) it follows that the acceleration of the particle is given by

$$
a(r)=-\omega^{2}[r-h(r)],
$$

where $\omega$ is a constant with dimension of angular frequency whose square is

$$
\omega^{2}=\frac{g}{R-h(R)},
$$

where $g=G M / R^{2}$ is the acceleration due to gravity on the surface of the Moon. From Eq. (3) and Eq. (6), it is found that at the center of the Moon, the acceleration vanishes while the velocity reaches its maximum value. Although the motion is periodic, it is not a SHM. Thus, the value of $\omega$ does not match the actual frequency of the motion. In the special case where the density is uniform and equal to $\rho_{c}$, the function $h(r)$ is null along the entire trajectory, as well as the constant $h(R)$. Therefore, considering such restrictions on Eq. (5), Eq. (6) and Eq. (7), the particle ends with SHM whose angular frequency is $\omega=\sqrt{g / R}$. The period $(T)$ of the motion (which is twice the crossing time), the maximum velocity $\left(v_{c}\right)$ reached by the particle and the maximum pressure $\left(P_{c}\right)$ exerted on the walls of the tunnel will be obtained, employing the conventional SHM formulas, namely:

$$
T=2 \pi \sqrt{\frac{R}{g}}, \quad v_{c}=\sqrt{g R}, \quad P_{c}=\frac{\bar{\rho} g R}{2} .
$$

In general, the Eq. (1) refers to a second-order nonlinear differential equation

$$
\ddot{r}(t)=a(r(t)), \quad r(0)=R, \quad \dot{r}(0)=0,
$$

whose solution gives us the position $r(t)$, the velocity $\dot{r}(t)$ and the acceleration $\ddot{r}(t)$ of the particle as a function of time. From these equations, we can numerically infer the ellapsed time of particle motion.

With an analysis based on the hydrostatic equilibrium, it is found that the pressure gradient inside the Moon is given by $d P / d r=\rho(r) a(r)$, where $a(r)$ is negative, vide Eq. (1). This expression allows us to infer that we will have a minimum pressure on the surface of the Moon where the density $\rho(R)$ vanishes and a maximum pressure $P_{c}$ in the center of the Moon where the acceleration $a(0)$ is null. Then, considering that the (atmospheric) pressure on the surface of the Moon is negligible, the pressure inside the Moon can be obtained by integration

$$
P(r)=\int_{R}^{r} \rho\left(r^{\prime}\right) a\left(r^{\prime}\right) d r^{\prime} .
$$

The proper quantity for the measure of susceptibility of a rigid body to the rotational motion is the moment of inertia, the smaller its value, the greater will be the ability of it to rotate. For a spherically symmetrical mass distribution, the moment of inertia can be calculated by

$$
I=\frac{8 \pi}{3} \int_{0}^{R} \rho(r) r^{4} d r
$$

which will be used in the Section 4 in order to get an analytic expression for the density. 


\section{VPREMOON model}

According to the VPREMOON model [8], we can subdivide the interior of the Moon into four layers with their corresponding densities:

$$
\rho(r)=\left\{\begin{array}{ccc}
\rho_{c} & \text { for } \quad \begin{array}{l}
0 \leq r \leq \alpha_{1}=0.380 \mathrm{Mm} \\
(\text { Core })
\end{array} \\
\rho_{2}(r) \quad \text { for } \quad \begin{array}{l}
\alpha_{1} \leq r \leq \alpha_{2}=1.6971 \mathrm{Mm} \\
(\text { Mantle) }
\end{array} \\
\rho_{3} & \text { for } \quad \begin{array}{l}
\alpha_{2} \leq r \leq \alpha_{3}=1.7361 \mathrm{Mm} \\
\text { (Lower crust) } \\
\rho_{4}
\end{array} \text { for } \begin{array}{l}
\alpha_{3} \leq r \leq R=1.7371 \mathrm{Mm} \\
\text { (Upper crust) }
\end{array}
\end{array}\right.
$$

where $\rho_{c}=5.171 \mathrm{~g} / \mathrm{cm}^{3}, \rho_{3}=2.762 \mathrm{~g} / \mathrm{cm}^{3}$ e $\rho_{4}=$ $2.600 \mathrm{~g} / \mathrm{cm}^{3}$. In Table 6 of the Ref. [8], the values of density associated with the mantle and the corresponding positions within the Moon have a correlation between them which can be described with good approximation through the quadratic polynomial $\rho_{2}(r)$ expressed in the sequel,

$\rho_{2}(r)=\left(3.44648+0.0054 \frac{r}{\mathrm{Mm}}-0.04894 \frac{r^{2}}{\mathrm{Mm}^{2}}\right) \mathrm{g} / \mathrm{cm}^{3}$. (12)

Then we can use the Heaviside step function to embrace the various layers and the density renders to

$$
\begin{aligned}
\rho(r)= & \rho_{c} \cdot\left[1-\theta\left(r-\alpha_{1}\right)\right]+\rho_{2}(r) \cdot\left[\theta\left(r-\alpha_{1}\right)\right. \\
& \left.-\theta\left(r-\alpha_{2}\right)\right]+\rho_{3} \cdot\left[\theta\left(r-\alpha_{2}\right)-\theta\left(r-\alpha_{3}\right)\right] \\
& +\rho_{4} \cdot\left[\theta\left(r-\alpha_{3}\right)-\theta(r-R)\right] .
\end{aligned}
$$

In Table 1 given below, the positions $(r)$ of the free falling particle in the first column are taken on the boundaries between the layers inside the Moon. The respective time $(t)$ and velocity $(v)$ - in the second and third columns - were provided by the numerical solution, obtained through a software that employs Runge-Kutta algorithm, of the differential equation and boundary conditions expressed in Eq. (9). The corresponding acceleration $(a)$ and pressure $(P)$ on the tunnel walls fourth and fifth columns - were respectively obtained from Eq. (1) and Eq. 10.

\section{Jing2014 model}

The VPREMOON model is not the most updated model. In 2014 it was proposed the model [9] which presents two

Table 1: Some physical quantities at the boundaries of the layers inside the Moon (VPREMOON).

\begin{tabular}{lcccc}
\hline$r(\mathrm{Mm})$ & $t(\mathrm{~s})$ & $v(\mathrm{~km} / \mathrm{s})$ & $a\left(\mathrm{~m} / \mathrm{s}^{2}\right)$ & $P(\mathrm{GPa})$ \\
\hline$R=1.7371$ & 0 & 0 & 1.62480 & 0 \\
$\alpha_{3}=1.7361$ & 35.0849 & 0.0570029 & 1.62449 & 0.00422408 \\
$\alpha_{2}=1.6971$ & 222.089 & 0.359553 & 1.60757 & 0.178292 \\
$\alpha_{1}=0.380$ & 1382.51 & 1.68622 & 0.549359 & 4.76574 \\
center $=0$ & 1602.58 & 1.74702 & 0 & 5.30548 \\
\hline
\end{tabular}

variants, the preferred one, which we will call JING1, and the other whose values of the thicknesses of the different layers are in parenthesis in Table 3 of the Ref. [9], which we will call JING2; in our text, we will keep the JING2 data in parenthesis.

Although there are more recent papers with more updated database for the Moon [10 12], we will employ the database contained in Table 3.13 of the Ref. [13]. Based on some articles [9,13 19, we can subdivide the interior of the Moon into four layers with their corresponding densities:

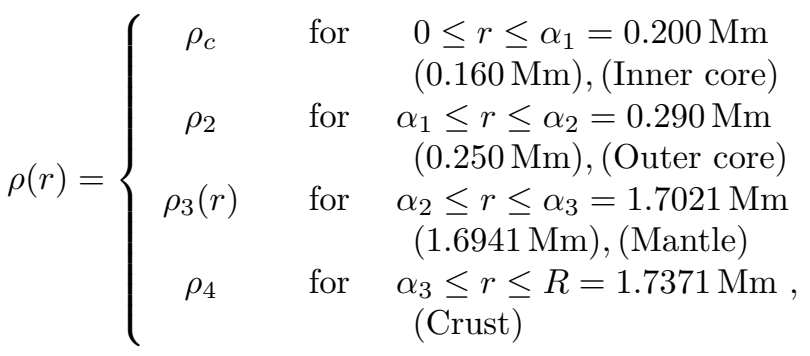

where $\rho_{c}=7.750 \mathrm{~g} / \mathrm{cm}^{3}, \rho_{2}=6.500 \mathrm{~g} / \mathrm{cm}^{3}$ and $\rho_{4}=$ $2.700 \mathrm{~g} / \mathrm{cm}^{3}$ for both variants of the model. For the mantle, quadratic approximations for $\rho_{3}(r)$ tied to the data in Table 3 of the Ref. [9] can be obtained from the following polynomial:

$$
\rho_{3}(r)=\rho_{\ell}+a\left(r-\alpha_{2}\right)+b\left(r-\alpha_{2}\right)^{2} .
$$

Three independent equations, each one containing this polynomial, are required to solve the unknowns $\rho_{\ell}, a$ and $b$. These equations are set forth in the sequel:

$$
\begin{aligned}
4 \pi \int_{0}^{R} \rho(r) r^{2} d r & =M, \\
\frac{8 \pi}{3 M R^{2}} \int_{0}^{R} \rho(r) r^{4} d r & =\frac{I}{M R^{2}}, \\
\rho_{3}\left(\alpha_{3}\right) & =\rho_{u} .
\end{aligned}
$$

Values of the quantities $M=73.477 \mathrm{Yg}\left(1 \mathrm{Yg}=10^{21} \mathrm{~kg}\right)$ 13, 15], $I / M R^{2}=0.3935,13,16$ and $\rho_{u}=3.32 \mathrm{~g} / \mathrm{cm}^{3}$ $\left(3.33 \mathrm{~g} / \mathrm{cm}^{3}\right)$ as are shown in Table 3 of the Ref. 9]. In this way, we solve the polynomials read, respectively, in JING1 and JING2 as

$$
\begin{aligned}
\rho_{3}(r) & =\left(3.468596960-0.03663021299 \frac{r}{\mathrm{Mm}}\right. \\
& \left.-0.02977009353 \frac{r^{2}}{\mathrm{Mm}^{2}}\right) \mathrm{g} / \mathrm{cm}^{3}, \\
\rho_{3}(r) & =\left(3.443579915+0.04189009170 \frac{r}{\mathrm{Mm}}\right. \\
& \left.-0.06430209456 \frac{r^{2}}{\mathrm{Mm}^{2}}\right) \mathrm{g} / \mathrm{cm}^{3} .
\end{aligned}
$$

The value of $\rho_{\ell}$ for each variant can be obtained with $\rho_{\ell}=\rho_{3}\left(\alpha_{2}\right)$. Then, from the point of view of the surface of the Moon, the mantle begins with density $\rho_{u}$ and ends with $\rho_{\ell}=3.455 \mathrm{~g} / \mathrm{cm}^{3}\left(3.450 \mathrm{~g} / \mathrm{cm}^{3}\right)$. 
Therefore, we embraces the various Moon layers, using Heaviside step function, and represent the density as

$$
\begin{aligned}
\rho(r) & =\rho_{c} \cdot\left[1-\theta\left(r-\alpha_{1}\right)\right]+\rho_{2} \cdot\left[\theta\left(r-\alpha_{1}\right)-\theta\left(r-\alpha_{2}\right)\right] \\
& +\rho_{3}(r) \cdot\left[\theta\left(r-\alpha_{2}\right)-\theta\left(r-\alpha_{3}\right)\right]+\rho_{4} \cdot\left[\theta\left(r-\alpha_{3}\right)\right. \\
& -\theta(r-R)] .
\end{aligned}
$$

The results set forth in Table 2 and Table 3 given below were obtained through the procedure used before to obtain the results in Table 1

\section{Comparing the different models}

We can compare the different models treated in the text in Table 4, namely:

Note that this table encloses the values that some quantities assume in the center of the Moon and also the time interval that a free falling particle takes to cross the entire tunnel drilled along a diameter of the Moon. To our surprise, this time interval is longer than $38.19 \mathrm{~min}$, which is the time interval that a particle would take to cross a tunnel drilled along a diameter through the Earth [4]. We also include the results for SHM, obtained from Eq. (8), where density takes the constant value $\bar{\rho}$ inside the Moon.

The three graphics below, Fig. 1, Fig. 2 a and Fig. $2 \mathrm{p}$ are about the kinematic of a free falling particle in the tunnel drilled along a diameter in the Moon.

The next two ones, Fig. 3a, Fig. 3 b, are about the inner structure of the Moon.

From Fig. 1 to Fig. 3b and Table 4 we can infer that the motion is composed of four parts associated to the four different layers and, except for the part associated with the mantle, the motions are precisely SHM with very different angular frequencies. In the particular case of the mantle, starting from the radius $0.380 \mathrm{Mm}$, all the curves of the Moon models treated in this text gradually approach to the SHM curve with angular frequency equal to $\sqrt{g / R}$. This approximation holds up to the radius $1.69 \mathrm{Mm}$ which is very close to the radius of the Moon.

Table 2: Some physical quantities at the boundaries of the layers inside the Moon (JING1).

\begin{tabular}{lcccc}
\hline$r(\mathrm{Mm})$ & $t(\mathrm{~s})$ & $v(\mathrm{~km} / \mathrm{s})$ & $a\left(\mathrm{~m} / \mathrm{s}^{2}\right)$ & $P(\mathrm{GPa})$ \\
\hline$R=1.7371$ & 0 & 0 & 1.62477 & 0 \\
$\alpha_{3}=1.7021$ & 207.711 & 0.336506 & 1.61140 & 0.152903 \\
$\alpha_{2}=0.290$ & 1436.16 & 1.71146 & 0.560093 & 4.91747 \\
$\alpha_{1}=0.200$ & 1488.34 & 1.73729 & 0.433226 & 5.20605 \\
center $=0$ & 1602.38 & 1.76208 & 0 & 5.54180 \\
\hline
\end{tabular}

Table 3: Some physical quantities at the boundaries of the layers inside the Moon (JING2).

\begin{tabular}{lcccc}
\hline$r(\mathrm{Mm})$ & $t(\mathrm{~s})$ & $v(\mathrm{~km} / \mathrm{s})$ & $a\left(\mathrm{~m} / \mathrm{s}^{2}\right)$ & $P(\mathrm{GPa})$ \\
\hline$R=1.7371$ & 0 & 0 & 1.62477 & 0 \\
$\alpha_{3}=1.6941$ & 230.264 & 0.372856 & 1.60846 & 0.187677 \\
$\alpha_{2}=0.250$ & 1459.80 & 1.71044 & 0.477085 & 4.92411 \\
$\alpha_{1}=0.160$ & 1512.07 & 1.73175 & 0.346581 & 5.16292 \\
center $=0$ & 1603.90 & 1.74768 & 0 & 5.37780 \\
\hline
\end{tabular}

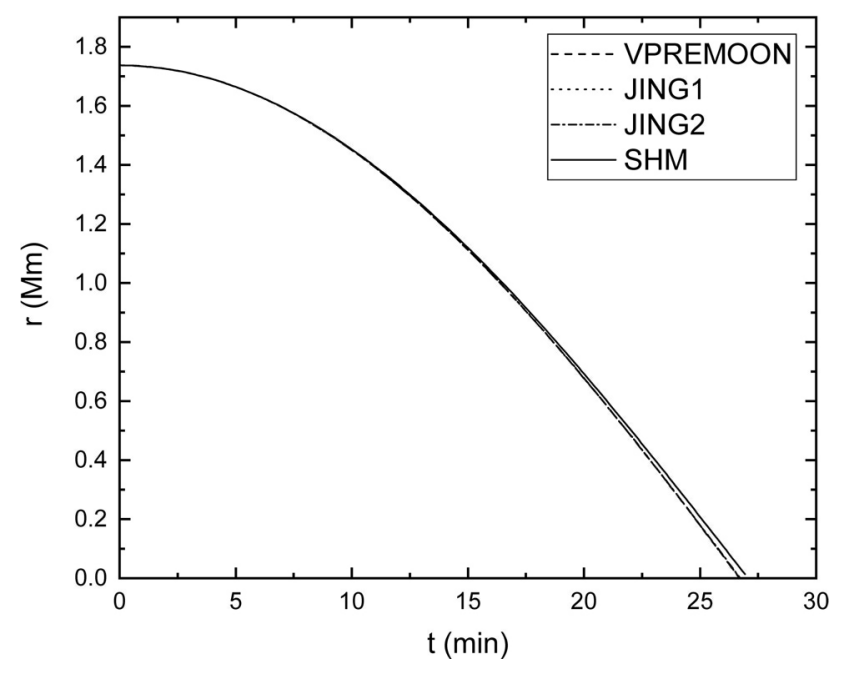

Figure 1: Position of the free falling particle as a function of time.

Then, we argue that the Moon model, based in constant density equals to average Moon density, it is a quite suitable approximation to SHM.

In order to put our work in a new perspective, we will compare the acceleration computed through Moon models with the one computed from the Earth model, which can be revealed through the graphics in Fig. 4a (Moon) and Fig. 4 b (Earth). The graphics (dashed curve) about the Earth were build through the density polynomials made available in Table I on PREM [5], with the same procedure employed for the Moon. The solid curves in both graphics refers to the constant density case (SHM)). With respect to Earth, with a more accurate analysis of the dashed curve given in the Fig. $4 \mathrm{~b}$, we find that the motion in the first 13.5 minutes is characterized by an approximately constant acceleration, around $10 \mathrm{~m} / \mathrm{s}^{2}$, and then during the remaining 5.6 minutes, the acceleration drops gradually until it reaches zero in the center of the Earth 20]. This result confirms what is advocated in the reference [4], which states that an Earth model based on constant acceleration is more realistic than that which is addressed in didactic literature in that the authors treat a planet as if it had constant density (SHM). For example, the PREM [5] gives us the realistic value 38.2 minutes for the crossing time while the SHM provides us 42.2 minutes. The model with constant acceleration, as can be read in the article 4], provides us with 38.0 minutes, a time that is practically identical to the PREM time. However, in the case of the Moon, we can verify that the constant density case (SHM) is the best approximation. For example, in Fig. 4a, we see that the curves for the Moon models treated in our text closely approximates the curve of the constant density model; as far as the crossing time, the Moon models provide us with the realistic values between 53.4 and 53.5 minutes, while the constant density model provides us with 54.1 minutes, which is quite close. Since the case of constant density is simpler to be addressed in the textbooks, its authors 


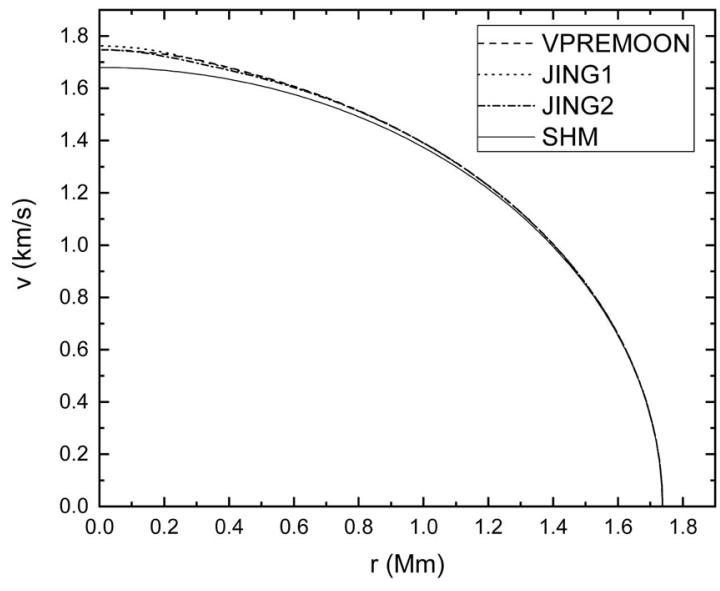

(a)

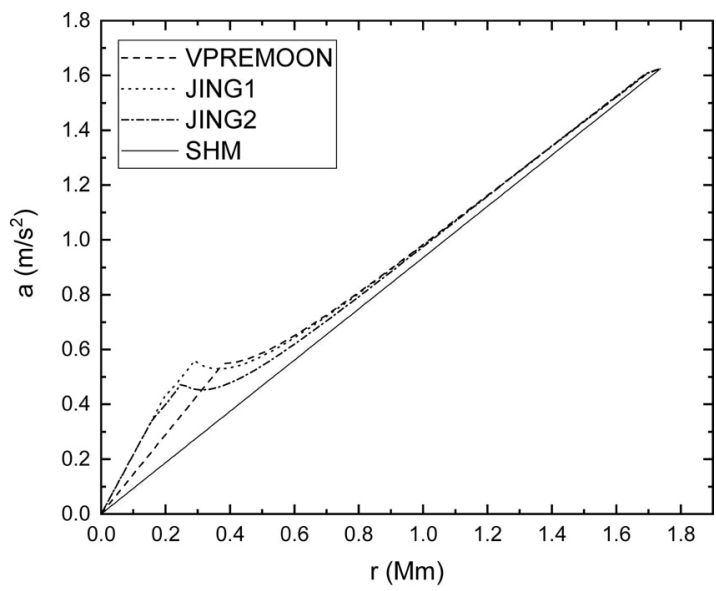

(b)

Figure 2: Velocity (2a) and Acceleration (2p) of the free falling particle as a function of position.

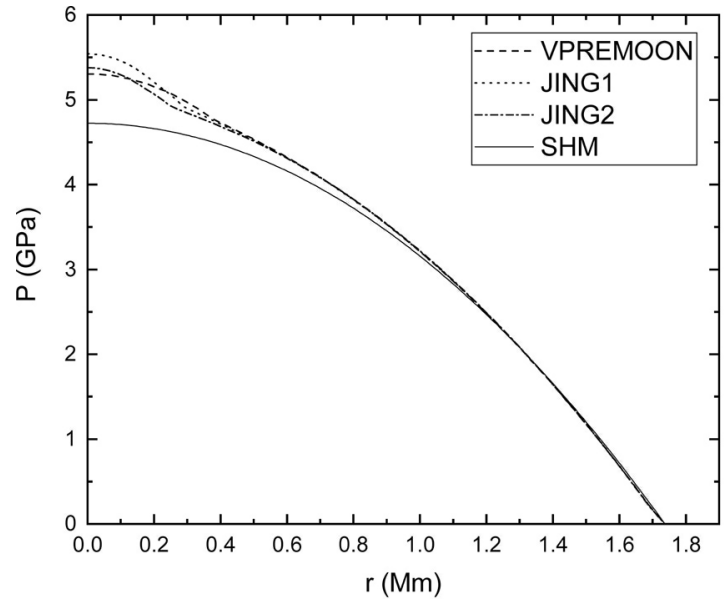

(a)

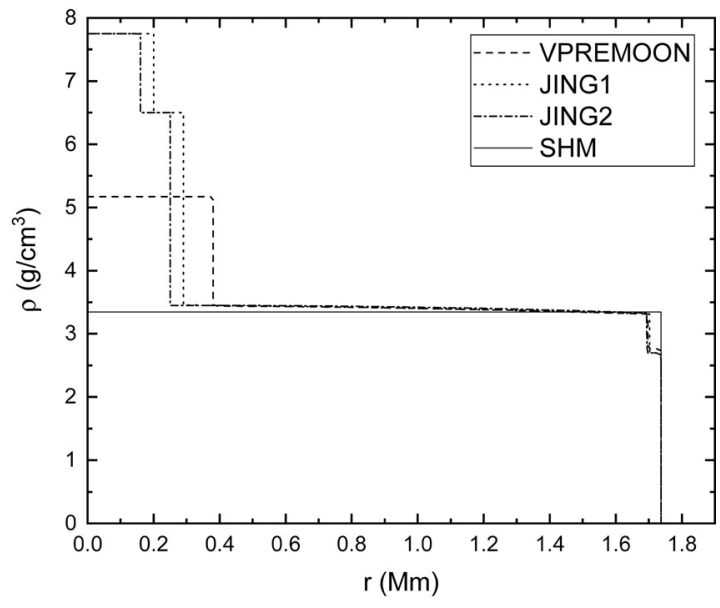

(b)

Figure 3: Pressure (3) and Density (3p) within the Moon as a function of the radius.

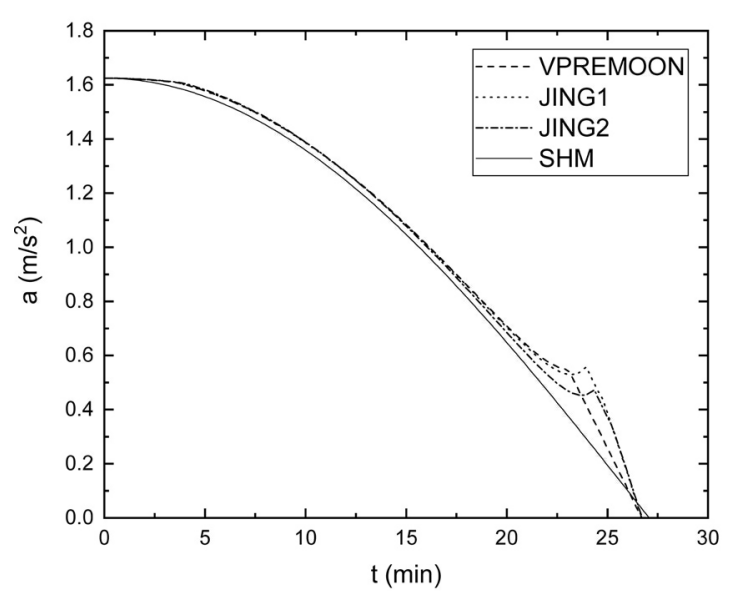

(a)

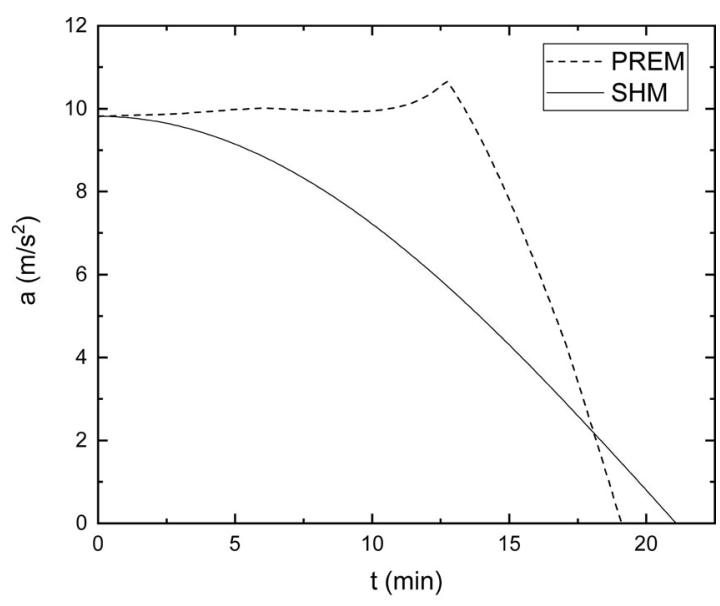

(b)

Figure 4: Acceleration of the free falling particle within the Moon $(4$ ) and Earth $(4 \mathrm{p})$ as a function of time. 
Table 4: Physical quantities associated with the gravitational tunnel through the Moon for various models.

\begin{tabular}{lcccc}
\hline Model & $\rho_{c}\left(\mathrm{~g} / \mathrm{cm}^{3}\right)$ & $v_{c}(\mathrm{~km} / \mathrm{s})$ & $P_{c}(\mathrm{GPa})$ & $t_{\text {crossing }}(\mathrm{min})$ \\
\hline VPREMOON & 5.171 & 1.747 & 5.305 & 53.42 \\
JING1 & 7.750 & 1.762 & 5.542 & 53.41 \\
JING2 & 7.750 & 1.748 & 5.378 & 53.46 \\
SHM & 3.346 & 1.680 & 4.723 & 54.14 \\
\hline
\end{tabular}

would come closer to reality if they considered the Moon instead of the Earth.

\section{Conclusions}

We have used some realistic models for the inner of the Moon and numerical procedures to calculate some physical features about the motion of free falling vehicles through tunnels inside that satellite. After that, we have compared those results with the correspondent ones computed when the Earth was considered, which confirms the results proposed by Klotz [4]. In his approach, based on an internal structure for our planet, the acceleration is assumed constant. It provides results much closer to the realistic ones than an approach based on constant density. However in the case of the Moon the exact opposite happens.

Usually, the physics textbooks at the undergraduate level must present simplifications, in such a way that problems can be solved quickly and in a few lines. Thus, they must have their approach based on constant density. Therefore, we have showed that Moon is more suitable to address this problem in these textbooks. It is interesting that future authors keep in mind that the Moon fits much more into this simplification than the Earth.

Another result that at first sight draws attention is that the realistic time $(53.4 \mathrm{~min})$ for crossing tunnels through the Moon is much greater than the realistic time (38.2 min) for crossing tunnels through the Earth, although the diameter of the Moon is much smaller than the diameter of the Earth. However, this result from the perspective of gravitational potential energy is not a surprising one, since the gravitational potential energy in the Earth is much larger than in the Moon, which means that, under free fall, at the cost of reducing gravitational potential energy, a vehicle would be considerably more accelerated within the Earth than within the Moon.

\section{Acknowledgments}

The authors would like to thank the reviewer for the time and effort required to review the manuscript as well as for the valuable comments and suggestions.

\section{References}

[1] J.E. Prussing, Am J Phys 44, 304 (1976).

[2] A. Redier, La Nature 520, 386 (1883).

[3] P.W. Cooper, Am J Phys 34, 68 (1966).

[4] A.R. Klotz, Am J Phys 83, 231 (2015).
[5] A.M. Dziewonski and D.L. Anderson, Phys Earth Planet Inter 25, 297 (1981).

[6] W.D. Pesnell, Am J Phys 84, 192 (2016).

[7] S. Chandrasekhar, in: An Introduction to the Study of Stellar Structure (Dover Publications, New York, 2012).

[8] R.F. Garcia, J. Gagnepain-Beyneix, S. Chevrot and P. Lognonné, Phys Earth Planet Inter 202-203, 89 (2012).

[9] Z. Jing, Y. Wang, Y. Kono, T. Yu, T. Sakamaki, C. Park, M.L. Rivers, S.R. Sutton and G. Shen, Earth Planet Sci Lett 396, 78 (2014).

[10] C. Hirt and W.E. Featherstone, Earth Planet Sci Lett 329-330, 22 (2012).

[11] S. Goossens, K. Matsumoto, Q. Liu, F. Kikuchi, K. Sato, H. Hanada, Y. Ishihara, H. Noda, N. Kawano, N. Namiki et al., J Geod 85, 205 (2011).

[12] D.E. Smith, M.T. Zuber, G.A. Neumann, F.G. Lemoine, E. Mazarico, M.H. Torrence, J.F. McGarry, D.D. Rowlands, J.W. Head III and T.H. Duxbury, Geophys Res Lett 37, L18204 (2010).

[13] M.A. Wieczorek, B.L. Jolliff, A. Khan, M.E. Pritchard, B.P. Weiss, J.G. Williams, L.L. Hood, K. Righter, C.R. Neal, C.K. Shearer et al., Rev Mineral Geochem 60, 221 (2006).

[14] D.E. Smith, M.T. Zuber, G.A. Neumann and F.G. Lemoine, J Geophys Res 102, 1591 (1997).

[15] A.S. Konopliv, S.W. Asmar, E. Carranza, W.L. Sjogren and D.N. Yuan, Icarus 150, 1 (2001).

[16] A.S. Konopliv, A.B. Binder, L.L. Hood, A.B. Kucinskas, W.L. Sjogren and J.G. Williams, Science 281, 1476 (1998).

[17] A.S. Konopliv, A.B. Binder, L.L. Hood, A.B. Kucinskas, W.L. Sjogren and J.G. Williams, Science 339, 671 (2013).

[18] R.C. Weber, P.Y. Lin, E.J. Garnero, Q. Williams and P. Lognonné, Science 331, 309 (2011).

[19] T. Komabayashi and Y. Fei, J Geophys Res 115, B03202 (2010).

[20] M.A. De Andrade and L.G. Ferreira Filho, Rev Bras Ens Fis 40 e3302 (2018). 